\title{
PENDEO-EPITAXY - A NEW APPROACH FOR LATERAL GROWTH OF GALLIUM NITRIDE STRUCTURES
}

\author{
Tsvetanka S. Zheleva*, Scott A. Smith, Darren B. Thomson, Thomas Gehrke, Kevin J. \\ Linthicum, Pradeep Rajagopal, Eric Carlson, Waeil M. Ashmawi, and Robert F. Davis \\ Department of Materials Science and Engineering, \\ North Carolina State University, Box 7907, Raleigh, NC 27695-7907 \\ *Also with: Army Research Lab, 2800 Powder Mill Road, Adelphi, MD 20783 \\ Corresponding Author (E-mail: Tsvetanka_Zheleva@ncsu.edu)
}

Cite this article as: MRS Internet J. Nitride Semicond. Res. 4S1, G3.38 (1999)

\section{ABSTRACT}

A new process route for lateral growth of nearly defect free $G a N$ structures via Pendeoepitaxy is discussed. Lateral growth of GaN films suspended from $\{11 \overline{2} 0\}$ side walls of [0001] oriented GaN columns into and over adjacent etched wells has been achieved via MOVPE technique without the use of, or contact with, a supporting mask or substrate. Pendeo-epitaxy is proposed as the descriptive term for this growth technique. Selective growth was achieved using process parameters that promote lateral growth of the $\{1 \overline{2} 0\}$ planes of GaN and disallow nucleation of this phase on the exposed SiC substrate. Thus, the selectivity is provided by tailoring the shape of the underlying GaN layer itself consisting of a sequence of alternating trenches and columns, instead of selective growth through openings in $\mathrm{SiO}_{2}$ or $\mathrm{SiN}_{\mathrm{X}}$ mask, as in the conventional lateral epitaxial overgrowth (LEO).

Two modes of initiation of the pendeo-epitaxial GaN growth via MOVPE were observed:

Mode A - promoting the lateral growth of the $\{11 \overline{2} 0\}$ side facets into the wells faster than the vertical growth of the (0001) top facets; and Mode B - enabling the top (0001) faces to grow initially faster followed by the pendeo-epitaxial growth over the wells from the newly formed $\{11 \overline{2} 0\}$ side facets. Four-to-five order decrease in the dislocation density was observed via transmission electron microscopy (TEM) in the pendeo-epitaxial GaN relative to that in the GaN columns. TEM observations revealed that in pendeo-epitaxial GaN films the dislocations do not propagate laterally from the GaN columns when the structure grows laterally from the sidewalls into and over the trenches. Scanning electron microscopy (SEM) studies revealed that the coalesced regions are either defect-free or sometimes exhibit voids. Above these voids the PEGaN layer is usually defect free.

\section{INTRODUCTION}

Recent studies on selected area growth (SAG) and lateral epitaxial overgrowth (LEO) of GaN structures on 6H-SiC [1-6] and Sapphire [7-10] have shown that the laterally overgrown regions have four-to-five orders of magnitude lower densities of dislocations compared to the regions of vertical growth. During the selective growth the GaN structure grows vertically and 
laterally at the same time, but the vertical growth dominates during the growth from the windows in $\mathrm{SiO}_{2}$ (i.e. the growth rate of (0001) planes of $\mathrm{GaN}$ is higher than the growth rate of the $\mathrm{GaN}$ side facets), while the lateral growth dominates during the $\mathrm{GaN}$ growth over the $\mathrm{SiO}_{2}$ (i.e. the growth rate of the GaN side facets is higher than the growth rate of the (0001) planes). Thus, the reduction in the defect density is associated with the change in the growth direction from vertical during the initial stages of growth from the openings in the $\mathrm{SiO}_{2}$, to lateral over the $\mathrm{SiO}_{2}$ in the subsequent stages of the lateral epitaxial overgrowth. Our previous studies on the development of the side facets morphology of the LEO-GaN as a function of the growth time [5,6], pointed that while the crystallographic template for the conventional vertical growth are the (0001) GaN faces, the crystallographic template for the lateral overgrowth are either the vertical $\{11 \overline{2} 0\}$ side facets if the $\mathrm{SiO}_{2}$ stripes are oriented along $\langle 1 \overline{1} 00\rangle$ direction, or the $62^{\circ}$ inclined $\{1 \overline{1} 01\}$ facets when the stripe edges are oriented along $\langle 11 \overline{2} 0\rangle$ direction, see the schematics in Fig.1. However, if the crystallographic template for the lateral overgrowth are the side GaN facets, then lateral growth would be possible even without a $\mathrm{SiO}_{2}$ mask. Then, a question rises: how to eliminate the $\mathrm{SiO}_{2}$ mask and still to preserve the crystallographic templates for the vertical growth (the (0001) planes) as well as the crystallographic templates for the lateral growth - the side facets, $\{11 \overline{2} 0\}$ or the $\{1 \overline{101}\}$ planes, depending on the stripe orientation? The new approach for lateral growth of nearly defect free $\mathrm{GaN}$ structures, namely pendeo -epitaxy is an answer to the above question [11].

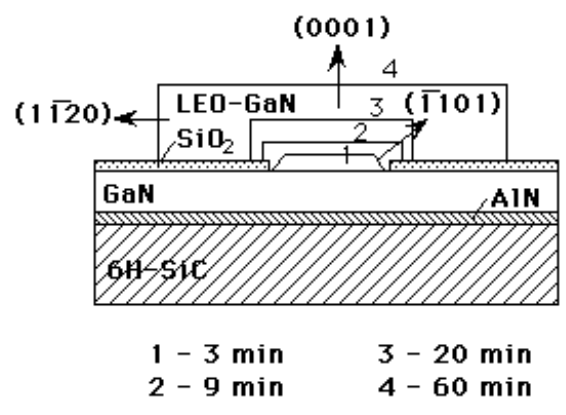

Figure 1. A schematic of the development of the side facet morphology of LEO-GaN structure as a function of the growth time in [1 $\overline{1} 00]$ orientation of the $\mathrm{SiO}_{2}$ stripes. The vertical $\{11 \overline{2} 0\}$ side facets are the crystallographic templates for the lateral growth.

\section{EXPERIMENT}

The new technique, namely, pendeo-epitaxy has been developed and employed for the lateral growth of $\mathrm{GaN}$ films from the side walls of sequential and parallel GaN columns [11]. An alternative approach employed in the present research to achieve lateral and vertical epitaxy of 
$\mathrm{GaN}$ is comprised of the deposition of this material upon an aligned series of $\langle 1 \overline{1} 00\rangle$ oriented columns or wells of GaN having the vertical $\{11 \overline{2} 0\}$ side facets, as shown schematically in Fig.2.
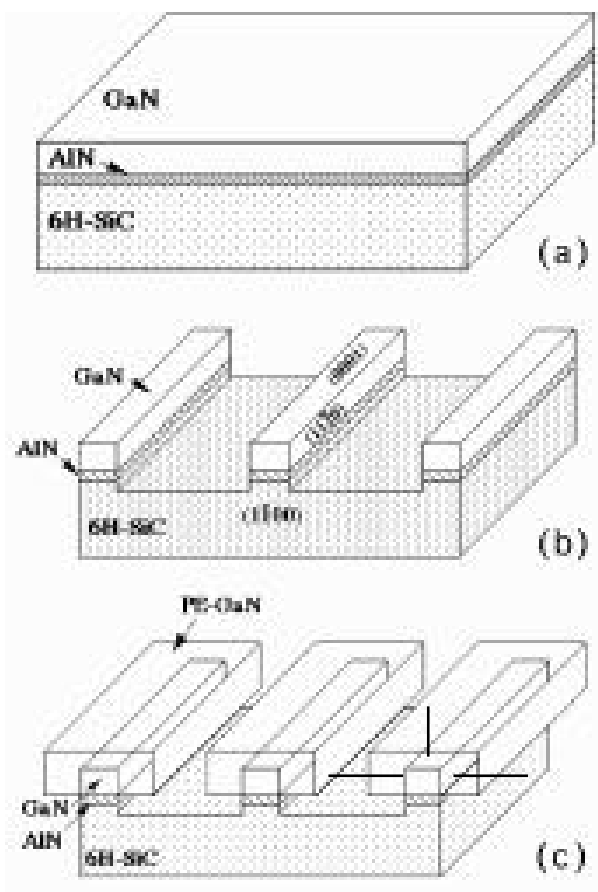

Figure 2. A schematic of the new process route for the pendeo-epitaxial growth of GaN layered structures. The PE-GaN growth includes the following major steps: (a) growth of a GaN layer on a AlN/6H-SiC substrate, (b) selective etching to form the side walls in the alternating GaN/AlN/substrate columns and trenches (wells), and (c) growth of the pendeo-epitaxial GaN layer before the coalescence.

The pendeo-epitaxial approach involves three major process steps: (i) growth of the underlying (seed) GaN layer on a suitable substrate, (ii) selective etching through a Ni mask [12] to form alternating GaN/AlN/substrate columns and trenches in the substrate, and (iii) growth of the pendeo-epitaxial GaN layer. No amorphous mask over the GaN seed layer is employed to support the laterally grown material and to separate it from a crystallographic surface. Thus, the lateral component of the GaN film grows free standing from the side walls of the columns into and over the etched trenches, as shown in Fig. 2(c). This laterally grown material does not make contact with the bottom surfaces of the trenches. As such, the term pendeo- (from the Latin "hangs on" or "suspends from") epitaxy was proposed to describe this process route [11]. Thus pendeo-epitaxy (PE), or the lateral growth from sidewalls of GaN posts into and over trenches provides an alternative process route to the conventional LEO technique, for growth of defect free GaN layers.

The side faces - crystallographic templates for the PE-GaN are prepared via etching of trenches (wells) or columns (posts), which include multiple sidewalls in the u-GaN/AlN/6H-SiC 
substrate-heterostructure. To form the columns and the trenches a dry etching technique with $\mathrm{Cl}_{2}$ and $\mathrm{Ar}$ as process gasses, and Ni mask in inductively coupled plasma (ICP) system were utilized. Details of the dry plasma etching parameters of GaN related with the etch rates and selectivities as a function of the ICP power, dc bias, the gases pressure and flow rates, as well as gases percentages have been previously investigated [12]. The PE-GaN growth was performed via metalorganic vapor phase epitaxy (MOVPE) using triethylgallium (TEG) and ammonia $\left(\mathrm{NH}_{3}\right)$ precursors, at $1080^{\circ} \mathrm{C}$ and 45 Torr. The TEG at $26 \mu \mathrm{mol} / \mathrm{min}$ and $\mathrm{NH} 3$ at $1500 \mathrm{sccm}$ were used in combination with $3000 \mathrm{sccm} \mathrm{H} 2$ diluent. The morphology and microstructure of the pendeo-epitaxial GaN films was studied with scanning electron microscopy (SEM-JEOL 6400-FE); and transmission electron microscopy (TEM-TOPCON 002B).

\section{RESULTS AND DISCUSSION}

Two modes of initiation of the pendeo-epitaxial GaN growth via MOVPE were observed, as shown schematically in Fig. 3, and pictorially in Fig. 4 (a-d): Mode A - promoting the lateral growth of the $\{11 \overline{2} 0\}$ side facets into the wells faster than the vertical growth of the (0001) top facets; and Mode B - enabling the top (0001) faces to grow initially faster followed by the pendeo-epitaxial growth over the wells from the newly formed $\{11 \overline{2} 0\}$ side facets. SEM studies revealed that the coalesced regions are either defect-free or sometimes exhibit voids. Above these voids the PE-GaN material is usually defect free. Preliminary SEM and TEM studies reveal four-to-five times larger lateral growth rates of the PE-GaN compared to the growth rates for the LEO-GaN for a similar geometry. Studies with AFM revealed atomically flat top and side surfaces for the PE-GaN material.A four-to-five order decrease in the dislocation density in the pendeo-epitaxial GaN relative to that in the GaN columns (dislocation density $\sim 10^{9} \mathrm{~cm}^{-2}$ ) was observed via TEM. TEM observations revealed that in pendeo-epitaxial GaN films the dislocations do not propagate laterally from the GaN columns when the structure grows laterally from the side walls into and over the trenches. The regions of coalescence of the pendeoepitaxial GaN structures are usually defect free above the voids.

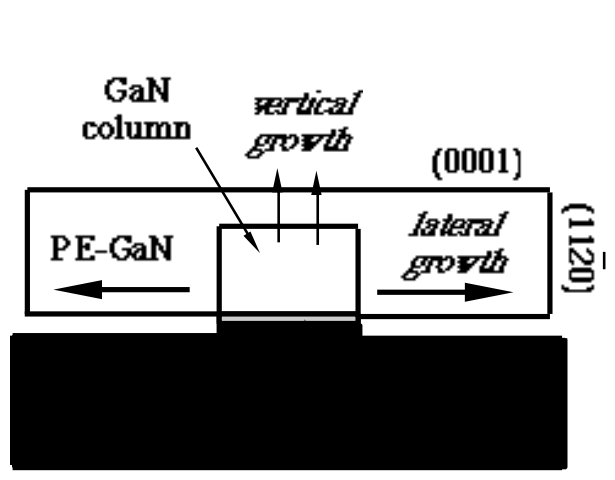

(a)

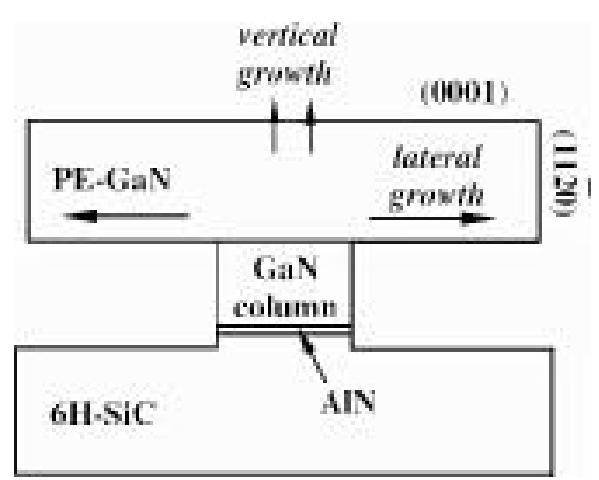

(b)

Figure 3. A schematic of the pendeo-epitaxial GaN growth in (a) Mode A and (b) Mode B. 


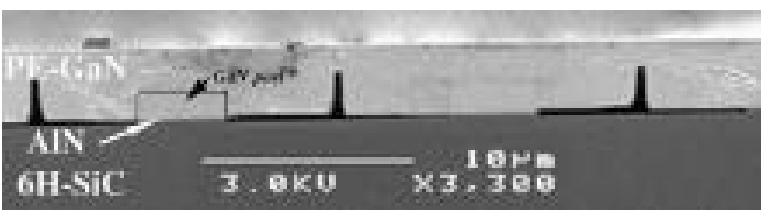

(a)

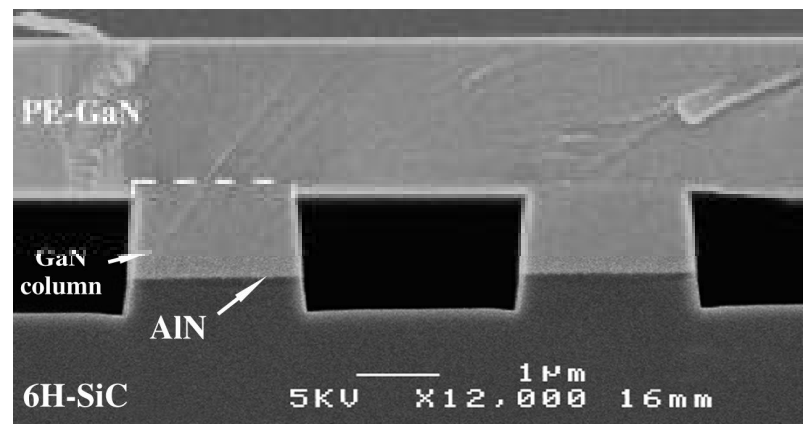

(c)

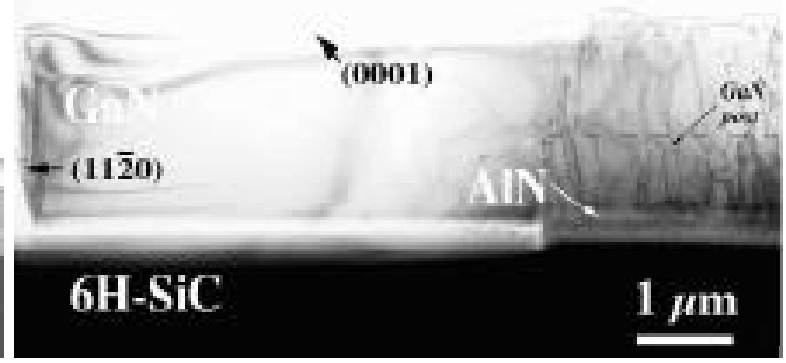

(b)

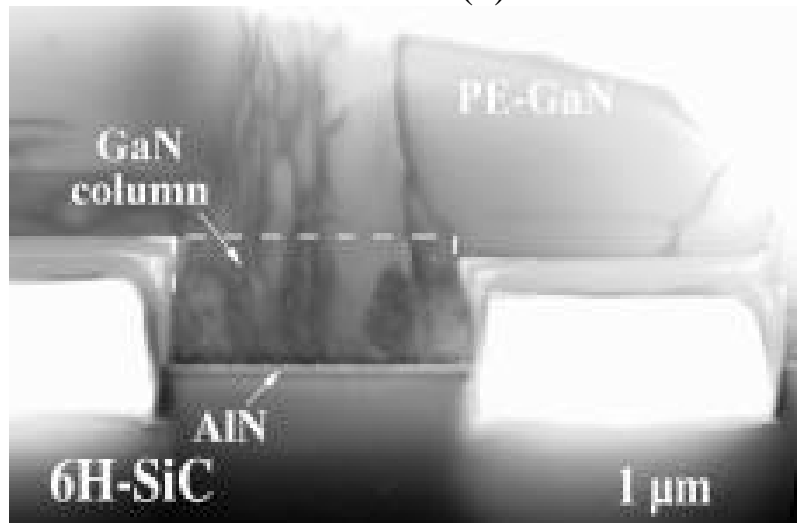

(d)

Figure 4. SEM (a) and TEM (b) micrographs of PE-GaN growth in Mode A; SEM (c) and TEM (d) of PE-GaN growth in Mode B.

Occasionally both growth modes are observed in a same column, i.e. on one side of the column - Mode A, and on the other side of the same column - Mode B. Our experiments revealed that slightly higher growth temperatures enhance the pendeo-epitaxial lateral growth from the side faces - Mode A. Thus differences in the morphology of the PE-GaN which appear as two different growth modes at same growth conditions are attributed to the fluctuations in the growth parameters as temperature and TEG flow rate, thus changing the diffusion characteristics. This suggests that there is a very narrow energy threshold associated with the parameters of the growth kinetics as mean free path and average life times until adsorption and incorporation of the adatoms, which enhances the growth either along [112 0] (Mode A), or along [0001] Mode B. Preliminary studies of the accommodation of the thermal stress in the PE-GaN structures reveal five-to-ten times reduction in the thermal stress compared to similar geometries of LEO-GaN structures.

Application of the pendeo-epitaxial growth technique in combination with a silicon nitride mask layer capping the seed GaN columns before the PE-GaN growth helps to achieve a continuous GaN layer of very low defects density within a single lithography step (see $\mathrm{K}$. Linthicum et all., D. Thomson et all., and T. Gehrke et all., this volume).

\section{SUMMARY}


A new technique - pendeo-epitaxy has been developed and employed for the lateral growth of GaN films from the side walls of sequential and parallel GaN columns. The new approach for the pendeo-epitaxial growth of GaN layered structures involves the following major stages: (a) growth of a GaN layer on a suitable substrate; (b) selective etching through a mask to form alternating GaN/AlN/substrate columns and trenches in the substrate, and (c) growth of the pendeo-epitaxial GaN layer. The pendeo-epitaxial GaN growth revealed two modes of growth at similar growth conditions: Mode A: PE-GaN growth from the side $\{11 \overline{2} 0\}$ walls of the GaN/AlN/substrate column into and over the wells, and Mode B: PE-GaN growth initiated from the (0001) planes followed by the lateral growth over the wells. Differences in the morphology of the PE-GaN layers are attributed to the fluctuations in the growth parameters as temperature and TEG flow rate, thus changing the diffusion characteristics. TEM studies revealed that the laterally grown regions exhibit four-to-five times lower dislocation density, compared to the regions within the GaN column and these defects do not propagate laterally into the PE-GaN regions. SEM and TEM studies revealed four-to-five times larger lateral growth rates of the PEGaN compared to the growth rates for the LEO-GaN for a similar geometry.

\section{ACKNOWLEDGMENTS}

The authors acknowledge Cree research, Inc. for the SiC wafers. This work was supported by the Office of Naval Research under contracts \# N00014-96-1-0765 (Colin Wood, monitor) and N00014-98-1-0654 (John Zolper, monitor). T. Zheleva acknowledges the National Research Council and National Academy of Sciences, and Dr. Kenneth A. Jones from the Army Research Lab, Adelphi, MD for the personal support.

\section{REFERENCES}

1. O.H. Nam, M.D. Bremser, B. Ward, R. J. Nemanich, and R.F. Davis, Jpn. J. Appl. Phys, 36, Part 2, No. 5A, L532 (1997)

2. T.S. Zheleva, O.H. Nam, M.D. Bremser, and R.F. Davis, Appl. Phys. Lett., 71, (17) 2472 (1997).

3. O.H. Nam, T.S. Zheleva, M.D. Bremser, and R.F. Davis, Appl. Phys. Lett., 71, (18), 2638 (1997).

4. T.S. Zheleva, O.H. Nam, J.D. Griffin, M.D. Bremser, and R.F. Davis, MRS Symp. Proc., 482, 393 (1997).

5. O.H. Nam, T.S. Zheleva, M.D. Bremser, and R.F. Davis, J. Electr. Mater., 27, No.4, 233 (1998).

6. T. Zheleva, O.H. Nam, W.M. Ashmawi, J.D. Griffin, R.F. Davis (submitted to J. Vac. Sci. \& Technol).

7. A.Usui, H. Sunakawa, A. Sakai, A.A. Jamaguchi, Jpn. J. Appl. Phys. 36, L899 (1997).

8. A.Sakai, H. Sunakawa, A. Usui, Appl. Phys. Lett. 71, 2259 (1997).

9. H. Marchand, J.P. Ibbetson, P.T. Fini, P. Kosodoy, S. Keller, S. denBaars, J.S. Speck, U.K. Mishra, Materials Internet Journal - Nitride Semiconductor Research, 3, 3 (1998).

10. Zhong H.Y., M.A.L. Johnson, T. McNulty, J.D. Brown, J.W. Cook Jr., J.F. Schezina, Materials Internet Journal -Nitride Semiconductor Research, 3, 6 (1998).

11. T. Zheleva, S. Smith, D. Thomson, K. Linthicum, and R.F. Davis (submitted to J. Electr. Mater.)

12. S.A. Smith, C. Wolden, M.D. Bremser, A.D. Hanser, R.F. Davis , W.V. Lampert, Appl. Phys. Lett., 71 (25), 3631 (1997). 